\title{
SUBCLAVIAN STEAL SYNDROME: A CASE REPORT
}

\author{
Meenakumari Ayekpam¹, Farooq Shafi², Tseizo Keretsu ${ }^{3}$
}

\section{HOW TO CITE THIS ARTICLE:}

Meenakumari Ayekpam, Farooq Shafi, Tseizo Keretsu. "Subclavian Steal Syndrome: A case report". Journal of Evolution of Medical and Dental Sciences 2014; Vol. 3, Issue 73, December 25; Page: 15461-15464, DOI: $10.14260 /$ jemds/2014/4085

ABSTRACT: Subclavian steal syndrome is a relatively rare condition that results from occlusion of the subclavian artery proximal to the origin of the vertebral artery. This causes a reversal in the direction of the blood flow in the vertebral artery, leading to symptoms of vertebrobasilar insufficiency. In this article, we report a case of subclavian steal syndrome in an elderly female, who was investigated with Doppler and CT angiography in our department.

KEYWORDS: subclavian steal, angiography, atherosclerosis.

CASE REPORT: A 60 year old female, a known hypertensive on medication, was admitted for evaluation of severe headache of 2 weeks duration, with mild tingling sensation in her left arm. The patient denied any history of chest pain, palpitation, visual changes, aura, nausea, fever or upper respiratory discharge.

Her medical history included long standing hypertension with hypercholesterolemia, and past history of left basal ganglia infarct.

On physical examination, her blood pressure was 140/90 mmHg (right arm), left radial pulse was feeble, following which BP was recorded in the left arm which was found to be markedly low at 80/44 mmHg. Other clinical findings, laboratory investigations, ECG and chest X-ray were unremarkable. CT brain showed a chronic infarct in the left basal ganglia. With the clinical suspicion of vertebral steal syndrome, the patient was referred for Doppler evaluation of the neck vessels.

Doppler study showed complete reversal of the blood flow in the left vertebral artery, both in systole and diastole (type 4 waveform) (Fig. 1); monophasic low velocity flow in the distal left subclavian artery; a non-occlusive soft plaque in the right carotid bulb and left internal jugular vein thrombosis. The proximal left subclavian artery was not accessible for the study. The findings were suggestive of Complete/Stage III subclavian steal syndrome and MDCT angiography of the neck vessels was advised.

MDCT angiography was performed following contrast injection, which showed irregular concentric wall thickening of the left subclavian artery near origin causing complete luminal stenosis. Bilateral vertebral arteries and the left subclavian artery distal to the origin of the Left Vertebral artery showed normal luminal opacification with contrast (Fig. 2), suggesting the feeding of distal left subclavian artery by the left vertebral artery. The left internal jugular vein thrombosis was also demonstrated. The findings were conclusive of subclavian steal syndrome secondary to occlusion of proximal left subclavian artery due to atherosclerosis.

DISCUSSION: Subclavian steal syndrome is relatively rare condition. Two times more common in females than in males, and patients are generally of the elderly age group. ${ }^{[1-2]}$ Subclavian steal syndrome occurs when the subclavian artery, most commonly the left, becomes occluded proximal to the origin of the vertebral artery. In this setting, the distal subclavian artery "steals" blood from the 
posterior circulation of the brain by means of retrograde flow through the ipsilateral vertebral artery. ${ }^{[3]}$ Decreased blood flow to the brain and upper extremity on the affected side results in a variety of symptoms, due to vertebrobasilar insufficiency or ischemia of the affected extremity.[1-2] Vertebrobasilar insufficiency may produce light-headedness, dizziness, vertigo, ataxia, visual disturbances, motor deficits, focal seizures, confusion, aphasia, headache, presyncope, syncope and rarely strokes. Symptoms due to ischemia of the affected extremity are less frequent and include weakness or arm claudication after exercise, paresthesias. The symptom can appear by vigorous exercise of the affected arm, and, or sudden turning of the head to the affected side.[1-2,4]

Arteriosclerosis is the most common cause (95\%). Other causes of occlusion include dissecting aortic arch aneurysm, embolism, and Takayasu's arteritis.

Physical findings of subclavian steal syndrome include unilaterally decreased pulses, $>20 \mathrm{~mm}$ Hg difference in blood pressure between the upper extremities, supraclavicular bruits, and disappearance of the radial pulse with exercise of the affected extremity.

Although subclavian steal is rare, a high index of suspicion is warranted in the presence of a suggestive history, risk factors, and physical findings. Differential diagnosis includes intracranial vascular disease, carotid artery disease, vertebral artery disease, brain tumor, and subdural hematoma. ${ }^{[2,4-5]}$ Diagnosis of subclavian steal syndrome ${ }^{[2]}$ is confirmed by carotid duplex ultrasonography, magnetic resonance or computed tomographic angiography. The Pulsed Doppler (PW) is useful in the analysis of the vertebral artery, recording information capable to indicate the presence of the subclavian steal syndrome. One of the main goals of the Doppler of vertebral arteries is the detection of reverse blood flow, indicating the phenomenon of subclavian steal.[6] There are four types of waveforms that indicate the degree of abnormal hemodynamics.[6]

Type 1: Flow velocity at the nadir of the mid-systolic notch greater than that of end-diastole.

Type 2: Velocity in mid-systolic notch equal to end-diastole.

Type 3: Velocity in mid-systolic notch at baseline.

Type 4: Velocity in mid-systolic notch below baseline.

Subclavian phenomenon is classified based on the degree of hemodynamic disturbances of the vertebral artery: stage I (Occult steal, decreased blood flow), stage II (partial steal, transient or partial reversal of flow), and stage III (Complete steal, permanent reversal of flow).[7]

The stenosis and vessel morphology can be assessed by CT or MR angiography. Time-resolved three-dimensional MRA has been described as a means of identifying reversal of vertebral artery flow. ${ }^{[8-9]}$ Phase contrast MRI can confirm the reversal of blood flow in the vertebral artery, and velocity-encoded cine phase contrast MRI can be used to quantify velocity and flow in the vertebral and subclavian arteries.[10] Conventional angiography, although invasive, can show stenotic or occlusive lesions in the subclavian, carotid, and vertebral arteries, as well as reversal of flow in the vertebral artery.

Risk factor modification (smoking cessation and control of hypertension, diabetes, and hypercholesterolemia) is essential.[2] Invasive treatment of subclavian steal syndrome is indicated in symptomatic patients. Options include axillo-axillary bypass, carotid-subclavian bypass, and percutaneous transluminal angioplasty (PTA) of the stenotic proximal subclavian artery with stent placement.[11,12] 


\section{CASE REPORT}

CONCLUSION: This case highlights the importance of subclavian steal syndrome, its wide range of clinical manifestations, and various modalities for its diagnosis. Recognition of this syndrome is crucial, since patients can be successfully treated with PTA and stent placement or other bypass graft operations.

FIG. 1: Showing complete reversal of flow (below the baseline) in both systole and diastole in left vertebral artery.

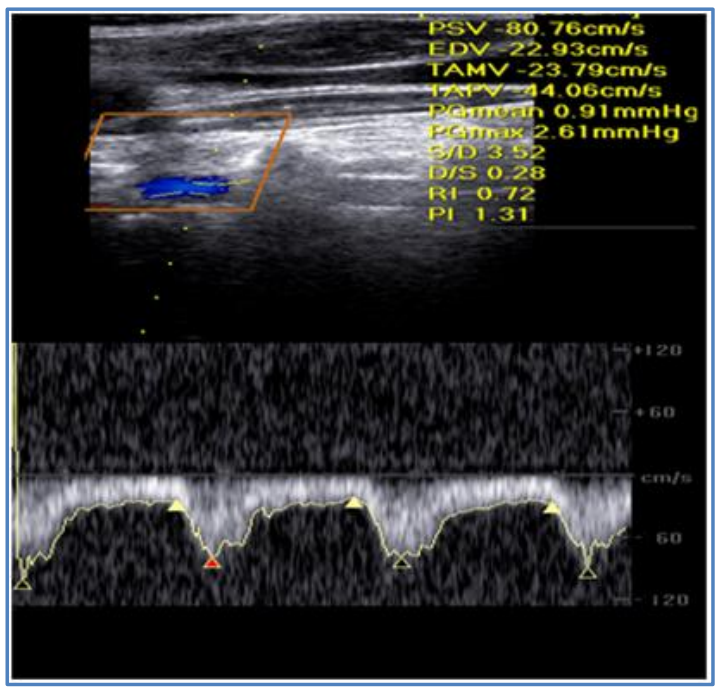

Fig. 1

FIG. 2: Coronal MIP reformatted image and a close up view showing irregular concentric wall thickening of the left subclavian artery near origin causing complete luminal stenosis (white arrows). Distal to the origin of Left Vertebral artery normal luminal opacification is noted.

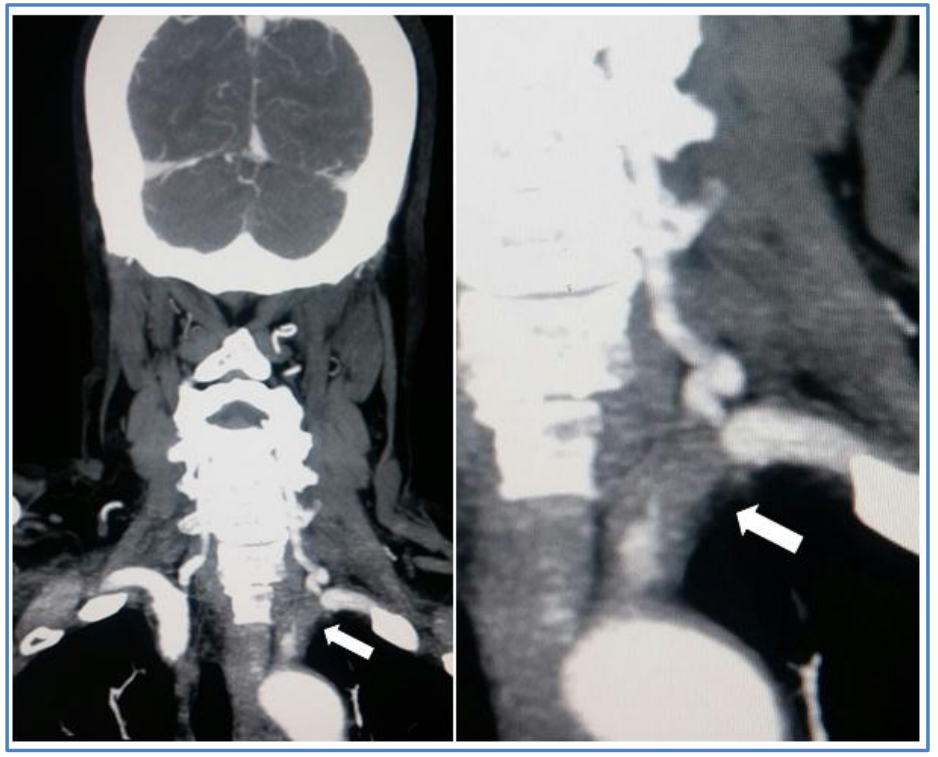

Fig. 2 


\section{REFERENCES:}

1. Reivich M, Holling HE, Roberts B. Reversal of blood flow through the vertebral artery and its effect on cerebral circulation. N Engl J Med 1961; 265: 878-85.

2. Harper CA, Haines JD. Subclavian steal syndrome: a report of two cases. Postgrad Med 1988; 83: 97-100.

3. Reivich M, Holling HE, Roberts B, et al. Reversal of blood flow through the vertebral artery and its effect on cerebral circulation. N Engl J Med 1961; 265: 878-85.

4. Sagkaguchi S: Syncope associated with exercise. Am J Cardiol 1995; 75: 476-81.

5. Kapoor W: Evaluation and outcome of patients with syncope. Medicine 1990; 69: 160-175.

6. Kliewer MA, Hertzberg BS, Kim DH, Bowie JD, Courneya DL, Carroll BA. Vertebral artery Doppler waveform changes indicating subclavian steal physiology. AJR. 2000; 174 (3): 815-9.

7. Branchereau A, Magnan PE, Espinoza H, Bartoli JM. Subclavian artery stenosis: hemodynamic aspects and surgical outcome. J Cardiovasc Surg 1991; 32: 604-12.

8. Schubert, R. Time-resolved, contrast-enhanced 3D MR angiography in the subclavian steal effect. Vasa 2010; 39 (1): 85-93.

9. Virmani R, Carroll TJ, Hung J, Hopkins J, Diniz L, Carr J. Diagnosis of subclavian steal syndrome using dynamic time-resolved magnetic resonance angiography: a technical note. Magn Reson Imaging 2008; 26 (2): 287-92.

10. Bauer AM, Amin-Hanjani S, Alaraj A, Charbel FT. Quantitative magnetic resonance angiography in the evaluation of the subclavian steal syndrome: report of 5 patients. J Neuroimaging 2009; 19: 250-52.

11. Stipa S, Cavallaro A, Sciacca V, Mingoli A, Sterpetti A, Di Marzo L et al. Cross-over axillary bypass. Treatment of subclavian or innominate artery obstructive lesions. Int Angiol 1987; 6 (4): 421-7.

12. Deriu GP, Milite D, Verlato F, Cognolato D, Frigatti P, Zaramella M et al. Surgical treatment of atherosclerotic lesions of subclavian artery: carotid-subclavian bypass versus subclaviancarotid transposition. J Cardiovasc Surg 1998; 39 (6): 729-34.

\section{AUTHORS:}

1. Meenakumari Ayekpam

2. Farooq Shafi

3. Tseizo Keretsu

\section{PARTICULARS OF CONTRIBUTORS:}

1. Associate Professor, Department of Radiodiagnosis, RIMS, Imphal.

2. Post Graduate Trainee, Department of Radiodiagnosis, RIMS, Imphal.

3. Post Graduate Trainee, Department of Radiodiagnosis, RIMS, Imphal.

\section{NAME ADDRESS EMAIL ID OF THE} CORRESPONDING AUTHOR:

Dr. Meenakumari Ayekpam,

Associate Professor,

Department of Radiodiagnosis,

RIMS, Imphal.

Email: meesharao@gmail.com

Date of Submission: 02/12/2014.

Date of Peer Review: 03/12/2014.

Date of Acceptance: 18/12/2014.

Date of Publishing: 24/12/2014. 